\title{
Monte-Carlo-Based Channel Characterization for Underwater Optical Communication Systems
}

\author{
Chadi Gabriel ${ }^{1,2}$, Mohammad-Ali Khalighi ${ }^{1}$, Salah Bourennane ${ }^{1}$, Pierre Léon $^{2}$, and Vincent Rigaud ${ }^{2}$
}

\author{
${ }^{1}$ Institut Fresnel, UMR CNRS 7249, Aix-Marseille Université, École Centrale Marseille, Marseille, France \\ 2 IFREMER, La Seyne Sur Mer, France \\ chadi.gabriel@ifremer.fr ; Ali.Khalighi@fresnel.fr ; Salah.Bourennane@fresnel.fr ; Pierre.Leon@ifremer.fr ; \\ Vincent.Rigaud@ifremer.fr
}

\begin{abstract}
:
We consider channel characterization for underwater wireless optical communication (UWOC) systems. We focus on the channel impulse response and, in particular, quantify the channel time dispersion for different water types, link distances, and transmitter/receiver characteristics, taking into account realistic parameters. We use the Monte Carlo approach to simulate the trajectories of emitted photons propagating in water from the transmitter towards the receiver. During their propagation, photons are absorbed or scattered as a result of their interaction with different particles present in water. To model angle scattering, we use the two-term Henyey-Greenstein model in our channel simulator. We show that this model is more accurate than the commonly used Henyey-Greenstein model, especially in pure sea waters. Through the numerical results that we present, we show that, except for highly turbid waters, the channel time dispersion can be neglected when working over moderate distances. In other words, under such conditions, we do not suffer from any inter-symbol interference in the received signal. Lastly, we study the performance of a typical UWOC system in terms of bit-error-rate using the simple on-off-keying modulation. The presented results give insight into the design of UWOC systems.
\end{abstract}

\section{Introduction}

Even though oceans and seas cover the majority of the earth surface, they are still generally unexplored. This is especially the case for the deep sea waters. The European FP7 SENSEnet project aims at developing novel sensors for underwater environment monitoring, as well as designing adequate infrastructures for the implementation and deployment of such sensors [2]. Of special interest are the underwater wireless sensor networks (UWSN) due to their flexibility and simplicity of deployment, compared to cabled networks.

An UWSN consists of spatially distributed autonomous nodes to which a number of sensors are connected. These nodes are linked together to exchange the data collected by the sensors. The network can be used for assessing the aqueous environment, monitoring the seafloor activity for disaster prevention (for example, surveillance of seismic activities in order to provide tsunami warnings), helping underwater geochemical prospecting, modeling the weather impact on the submarine life, etc. 
Concerning the communication link, special attention should be devoted to the underwater optical channel properties. Due to their strong attenuation in water, radio frequencies cannot be used, unless over ranges of a few centimeters [3]. Acoustic waves are traditionally used for establishing relatively long range wireless underwater links. However, they are limited in bandwidth and their celerity is very low (around $1500 \mathrm{~m} / \mathrm{s}$ ) leading to serious problems for realtime high-rate communication. Moreover, time synchronization is very difficult, external noise sources considerably affect acoustic signals, and the usually-used large antennas are highly energy consuming [4], [5], [6]. All these make the implementation of an acoustic underwater system problematic in our application.

Optical underwater communication turns to be an appropriate solution for communication over ranges up to several tens of meters thanks to its cost-effectiveness and low-energy consumption [7], [8], [9], [10]. Using a suitable wavelength (in the blue/green range), we can attain high datarates (up to 1 Gbps over a few meters as reported in [7]), depending on the water conditions and the transmitter/receiver parameters. This can enable data, image, and even video transmission between the nodes of an UWSN. However, optical communication in water is not an easy task since the optical beam is subject to strong intensity attenuation due to light absorption and scattering. In addition, scattering can create inter-symbol interference (ISI) by causing pulse stretching when transmitting with high data rates and over long distances [7], [11]. The induced ISI can degrade the quality of data transmission, and may necessitate computationally complex signal processing (i.e., channel equalization) at the receiver. So, an important step in the design of an UWSN is to accurately characterize the underwater optical channel by taking into account these phenomena. Based on an accurate channel model, one can set the system parameters appropriately in order to establish a high-quality link between the network nodes.

In this work, we consider comprehensive modeling of the underwater optical communication channel based on the Monte Carlo simulation method by which we simulate the trajectories of the emitted photons. We take into account different system parameters such as the transmitter beam width and beam divergence, beam wavelength, water type and turbidity, link distance, and the receiver field-of-view and aperture size. In particular, we use the two-term Henyey-Greenstein model for photon scattering which is a more accurate model than the simple Henyey-Greenstein 
one, considered in [1], [12]. We evaluate the impulse response of the optical channel under different conditions and show that, in most practical cases, the channel time dispersion can effectively be neglected. Also, for demonstration purposes, we consider a typical UWOC system and study its performance in terms of bit-error-rate (BER). For this, we consider the simple onoff-keying (OOK) modulation without channel coding, and illustrate how the BER performance is affected by the link distance and the water type.

The remainder of the paper is organized as follows. In Section II, we briefly present some previous works related to underwater optical propagation modeling while specifying our contributions with respect to them. In Section III, we recall the main characteristics of the water channel and the main equation governing light propagation in water. The description of our Monte Carlo simulator is provided in Section IV and modeling of photon scattering is discussed in Section V. In Section VI, we present some numerical results to identify the channel impulse response (IR) and to show the impact of different system parameters on the channel time dispersion. Also, we present in this section the BER performance for a simple case study. Finally, Section VII concludes the paper.

\section{PREVIOUS WORKS RELATED TO UNDERWATER OPTICAL COMMUNICATION}

Several recent works have considered channel effects in underwater wireless optical communication. Most of them neglect the channel dispersion due to scattering and use the simple exponential intensity attenuation model for optical beam propagation. In [8], [13], the performance of a wireless underwater optical communication in various water types and at different ranges is studied. In [10], the author considers, in particular, a modulating retro-reflector and a reflective link and shows that using the scattered light can improve the system performance in some special cases. In [3], the authors study the spatial and angular effects of scattering on a laser link based on the radiative transfer equation (RTE) and also present some laboratory experiments.

Two works that have particularly focused on channel time dispersion are those of [11] and [7]. In [11], the author uses the RTE with the modified Stokes vector to model light scattering in water. Considering polarized light, he studies the effect of the transmission distance on the 
channel time dispersion and concludes that ISI is very restrictive over long ranges $(50 \mathrm{~m})$ and at high rates (1 Gbps). However, the water parameters considered in [11] are far from most practical cases and correspond to a too dispersive medium: the average cosine of the scattering angle (see Section V) is set to 0.1348 and the ratio $b / c$ to 0.9767 (see Section III for the definitions of $b$ and $c$ ). The difference of our study with [11] is that, here, we take practical system parameters into account, particularly concerning the transmitter and the receiver, and also consider realistic water parameters. Also, we do not take into account light polarization because we consider intensity modulation with non-coherent detection, which is usually used in most systems due to its simplicity.

On the other hand, in [7], the authors present a laboratory experiment for a 1 Gbps-rate optical transmission system over a $2 \mathrm{~m}$ path length. They also present the channel transfer function by means of Monte Carlo simulations for longer transmission ranges and for different water types. Compared to [7], here we consider the channel impulse response as the main channel characteristic and quantify the channel time dispersion, especially, by considering different transmitter beam divergences and receiver aperture sizes. Moreover, we use the more accurate two-term Henyey-Greenstein model for simulating the trajectories of the scattered photons.

Several laboratory testbeds have also been developed for point-to-point or broadcast underwater optical communication. We provide here a brief presentation of some of them. An underwater sensor network called AquaNodes has been presented in [14], [15]. In this system, in order to reduce energy consumption and system cost, it is proposed to use acoustic links for broadcast communication over ranges up to $400 \mathrm{~m}$ (with a typical rate of $300 \mathrm{bps}$ ), and optical links for point-to-point communication between nodes over a range of $2 \mathrm{~m}$ (with a typical rate of $330 \mathrm{Kbps})$. Also, a system developed by WHOI has been introduced in [16] as a low-power, low-cost communication system, which uses a set of 22 red LEDs and supports transmission over a range of $5 \mathrm{~m}$ with a data-rate of up to $14.4 \mathrm{Kbps}$. Another prototype is presented in [17] which uses $2 \mathrm{~W}$ green/blue LEDs and allows directional transmission over a range of up to $5 \mathrm{~m}$ with a data rate of $57.6 \mathrm{Kbps}$. The AquaOptical system presented in [18] proposes, in particular, a high-rate system for medium range transmission. However, the developed system is highly 
power consuming: it uses six $5 \mathrm{~W}$ LEDs for a data rate of $1 \mathrm{Mbps}$ over $25 \mathrm{~m}$. More recently, a low-cost optical UWSN has been presented in [19] that can work over moderate distances (typically $10 \mathrm{~m}$ ) but has a very low transmission rate (about $310 \mathrm{bps}$ ).

These already-developed systems are not really adequate for our application where we require a node separation on the order of tens of meters and a high transmission rate of more than $10 \mathrm{Mbps}$. The characterization of the underwater optical channel, that we consider in this paper, is hence an important step in the development of our UWOC system.

\section{Characteristics OF THE OPTICAL PROPAGATION CHANNEL}

\section{A. Effect of water on optical propagation}

The two main processes affecting light propagation in water are absorption and scattering, which are both wavelength dependent [6], [20], [21]. Absorption is the irreversible loss of intensity and depends on the water's index of refraction. The spectral absorption coefficient $a(\lambda)$, with $\lambda$ being the light wavelength, is the main intrinsic optical property (IOP) to model the water absorption. Scattering, on the other hand, refers to the deflection of light from its original path. In water, deflections can be caused by the particles of size comparable to the wavelength (diffraction), or by the particulate matters with refraction index different from that of the water (refraction). Figure 1 illustrates the propagation behavior of a light flux when encountering a particle. The spectral volume scattering function (VSF) $\beta(\Psi, \lambda)$ is defined as the fraction of incident power scattered out of the beam through an angle $\Psi$ around a solid angle $\Delta \Omega$ centered on $\bar{\Psi}$. The VSF is used as the main IOP to model scattering. Integrating the VSF over all directions, gives the spectral scattering coefficient $b(\lambda)$ :

$$
b(\lambda)=2 \pi \int_{0}^{\pi} \beta(\Psi, \lambda) \sin \Psi d \Psi .
$$

Another useful parameter is the back-scattering coefficient $b_{b}(\lambda)$ that is obtained by integrating the VSF in the range $[\pi / 2, \pi]$ :

$$
b_{b}(\lambda)=2 \pi \int_{\pi / 2}^{\pi} \beta(\Psi, \lambda) \sin \Psi d \Psi .
$$


Lastly, the spectral beam attenuation coefficient $c$ (also called the extinction coefficient) is defined as the sum of $a$ and $b$ :

$$
c(\lambda)=a(\lambda)+b(\lambda)
$$

Note that $a, b, b_{b}$, and $c$ are in units of $\mathrm{m}^{-1}$.

The performance of an UWOC system can also be affected by channel fading as a result of oceanic turbulence. This is similar to the atmospheric turbulence in free-space optical communication [22], [23]. Water turbulence is generally due to the variations of the water refractive index, caused by the changes in the temperature, salinity, and the pressure of the water [24]. In general, the effect of the pressure on the water refractive index can be neglected [24]. Also, deep seas have generally an approximately constant level of salinity and the temperature variations are usually very small. As a result, the channel fading due to water turbulence is negligible in most practical cases as shown in [25].

At last, we assume that in our application, i.e., in deep sea, there is no probable beam blockage caused by bubbles, fish, or large suspended particles.

\section{B. Particles in water}

In addition to wavelength, both absorption and scattering largely depend on the level of turbidity and the type of particles in solution and suspension in water [26]. The main particles we are concerned with are explained in the following.

- Various dissolved salts which increase the scattering effect.

- Detrital and mineral components such as ground quartz sand, clay minerals, and metal oxides, which affect both absorption and scattering.

- Colored dissolved organic matters (CDOM) such as fluvic and humic acids which affect absorption, especially for blue and ultraviolet wavelengths. Their effect is more pronounced at the water surface and in the estuaries.

- Organic matters such as viruses, colloids, bacteria, phytoplankton, zooplankton, and organic detritus. They contribute in general to backscattering, especially in the blue spectral range. 
The spectral absorption and scattering coefficients, $a$ and $b$, can be calculated by adding the contribution of each class of particles to the corresponding coefficients of the pure sea water. Organic particles and especially phytoplanktonic matters play an important role in the optical properties of most oceanic waters. In fact, their chlorophyll pigments strongly absorb the light in the blue and red spectral ranges. These particles effectively determine the absorbance of the sea water and strongly contribute to the scattering coefficient [12], [27]. Therefore, one can use the chlorophyll concentration $C$ (in $\mathrm{mg} \cdot \mathrm{m}^{-3}$ ) as the free parameter to compute $a$ and $b$, based on bio-optical models such as those proposed by Haltrin and Kattawar [28], [27] or Gordon and Morel [12].

Let us explicitly see the impact of $C$ on the absorption and scattering properties of water. We have shown in Fig. 2 curves of $a, b$, and $c$, as a function of $\lambda$ using the model of Gordon and Morel for two chlorophyll concentrations of 0.31 and $0.83 \mathrm{mg} \cdot \mathrm{m}^{-3}$. We notice that an increase in $C$ has negligible impact on $a$ but it considerably affects $b$.

\section{Water types}

Knowing that underwater matters and water quality are variant from one region to another, four major water types are usually considered in the literature [3], [7], [29]:

- Pure sea waters: Absorption is the main limiting factor. The low $b$ makes the beam propagate approximately in a straight line.

- Clear ocean waters: They have a higher concentration of dissolved particles that affect scattering.

- Coastal ocean waters: They have a much higher concentration of planktonic matters, detritus, and mineral components that affect absorption and scattering.

- Turbid harbor and estuary waters: They have a very high concentration of dissolved and in-suspension matters.

Table I shows the typical values of the parameters $a, b, b_{b}$, and $c$, associated with these water types [3] that we will consider hereafter. 


\section{Light propagation in water}

The behavior of the light radiance in a propagation medium is described by the radiative transfer equation (RTE), given the medium IOP properties and the light beam characteristics. Let us denote by $L(z, \theta, \phi, \lambda)$ the light radiance in units of $\mathrm{Wm}^{-2} \mathrm{sr}^{-1} \mathrm{~nm}^{-1}$, with $z$ being the distance from the transmitter, and $\theta$ and $\phi$ the polar and azimuthal angles, respectively. Let us define the parameter $r$ as $r=z / \cos \theta$. We have [26], [30]:

$$
\frac{d L}{d r}=-c L+L^{\mathrm{E}}+L^{\mathrm{I}} \quad\left(\mathrm{Wm}^{-3} \mathrm{sr}^{-1} \mathrm{~nm}^{-1}\right)
$$

where $L^{\mathrm{E}}$ and $L^{\mathrm{I}}$ denote path functions for elastic and inelastic scattering, respectively. Inelastic scattering corresponds to the loss of photons due to a wavelength change. Because of its relatively low contribution to the general solution of the RTE, we neglect inelastic scattering. Note that most previous works neglect the $L^{E}$ term, (i.e., scattering) and consider straight-line propagation, described by the simple Lambert's law:

$$
L(z)=L(0) \exp (-c z)
$$

In this paper, we do take scattering into account but instead of solving (4), we use the Monte Carlo method as described in the following section.

\section{Channel modeling Based on Monte Carlo simulation}

We use Monte Carlo simulations based on the MCML method [31] to solve the RTE. Albeit its simplicity and flexibility, it is a rigorous approach for modeling photon transport in water. The main parameters that we take into consideration in our Monte Carlo simulator are:

- The transmitter characteristics, i.e., the wavelength, the beam width $w_{0}$, and the maximum initial divergence angle, denoted here by $\theta_{0, \max }$.

- The distance $Z$ between the transmitter and the receiver, and the medium described by the chlorophyll concentration $C$.

- The receiver characteristics, i.e., the aperture size and the field of view (FOV).

The simulator relies on the local probabilistic rules of photon propagation in water as explained in the following. 


\section{A. Initialization}

Initially, each photon is launched into the medium with a unity weight. Given the beam width $w_{0}$ and the maximum initial divergence angle $\theta_{0, \max }$, the initial position and the departure direction of the photon are determined based on three random variables (RVs). The position is generated according to $\mathcal{U}\left[0, w_{0}\right]$, and the direction according to $\mathcal{U}\left[-\theta_{0, \max }, \theta_{0, \max }\right]$ for $\theta$ and $\mathcal{U}[0,2 \pi]$ for $\phi$. Note that $\mathcal{U}[n, m]$ denotes the Uniform distribution between $n$ and $m$.

\section{B. Interaction of photon with a particle}

The considered emitted photon travels a distance $\delta$ (what we will refer to as the step size) before interacting with a particle in the medium. To generate $\delta$ randomly, we use a RV $\chi_{\delta}$ of distribution $\mathcal{U}[0,1]$, and calculate $\delta$ using (5) as follows [31].

$$
\delta=-\log \left(\chi_{\delta}\right) / c
$$

When interacting with the particle, the photon loses a fraction of its initial weight (what we will refer to as weight drop) and is deviated from its initial direction (photon scattering). Let us denote the photon weight before and after the interaction by $W_{\text {pre }}$ and $W_{\text {post }}$, respectively. We have [26]:

$$
W_{\text {post }}=W_{\text {pre }}(1-a / c) \text {. }
$$

Photon scattering is described in detail in Section $\mathrm{V}$, where we explain how we determine the new propagation direction of the photon (i.e., the new $\theta$ and $\phi$ ) after the interaction with a particle.

\section{Received photons}

This cycle of "step size generation $\rightarrow$ weight drop $\rightarrow$ angle scattering" is repeated until one of the following events happens:

- The photon weight is too small and negligible. The photon is considered as absorbed. This limit is set to $10^{-4}$ here.

- The photon reaches the receiver plane. If it is in the receiver aperture, it is considered as effectively received. Otherwise, it is considered as lost. 
Our simulator returns the proportion of absorbed, lost, and received photons' weights, as well as the Cartesian coordinates of the point of impact at the receiver plane. In addition, it calculates the total distance traveled by each photon until it reaches the receiver. This parameter can be converted to the propagation delay from the transmitter to the receiver.

Note that this method is statistical in nature and relies on calculating the propagation of a large number of photons. In our simulations, we have generated at least $10^{6}$ photons for each experiment, and have repeated the experiments at least $10^{3}$ times to obtain reliable results.

\section{Photon ANGLE SCATTERING}

We explain here how we have modeled photon scattering in our Monte Carlo simulator. As seen in the previous section, after interaction with a particle, the photon is deviated from its incoming direction. The new propagation direction is determined by regenerating randomly the azimuthal angle $\phi$ and the scattering angle $\theta$. Angle $\phi$ is considered as a RV of distribution $\mathcal{U}[0,2 \pi]$. The distribution of the scattering angle $\theta$, on the other hand, should take into account the medium characteristics. There are mainly two methods that are considered for modeling the distribution of $\theta$ : the simple Henyey-Greenstein and the two-term Henyey-Greenstein model.

\section{A. Henyey-Greenstein model}

Originally proposed for galactic scattering in Astrophysics by Henyey and Greenstein [32], the Henyey-Greenstein (HG) phase function defined by (8) is used in oceanic optics to model light scattering [12].

$$
p_{\mathrm{HG}}(\theta, g)=\frac{1-g^{2}}{2\left(1+g^{2}-2 g \cos \theta\right)^{3 / 2}} .
$$

Here, $g$ is the HG asymmetry parameter that depends on the medium characteristics and is equal to the average cosine of the scattering angle $\theta$ over all scattering directions, denoted by $\overline{\cos \theta}$. In other words, for the HG model, we take $g=\overline{\cos \theta}$. In fact, (8) describes the probability distribution of the deflection angle $\theta$. It is proposed in [26] to set $g=0.924$ as a 
good approximation for most practical situations. ${ }^{2}$

To randomly generate $\theta$, we first generate a $\mathrm{RV} \chi_{\mathrm{HG}}$ of distribution $\mathcal{U}[0, \pi]$, and then calculate the corresponding $\theta$ using the following equation.

$$
\chi_{\mathrm{HG}}=\int_{0}^{\theta} p_{\mathrm{HG}}(\Psi, g) \sin \Psi d \Psi
$$

\section{B. Two term Henyey-Greenstein model}

The interest of the HG function is its simplicity since it allows an easy computation of the RTE. However, it inadequately describes light scattering in water for small and large angles, namely for $\theta<20^{\circ}$ and $\theta>130^{\circ}$ [26]. A modified phase function, called the two-term HenyeyGreenstein (TTHG), has later been proposed in the literature [12], [35], that matches better the experimental results, e.g. those obtained by Petzold [34]. The TTHG function is given by:

$$
p_{\mathrm{TTHG}}\left(\theta, \alpha, g_{\mathrm{FWD}}, g_{\mathrm{BKWD}}\right)=\alpha p_{\mathrm{HG}}\left(\theta, g_{\mathrm{FWD}}\right)+(1-\alpha) p_{\mathrm{HG}}\left(\theta,-g_{\mathrm{BKWD}}\right),
$$

where $\alpha$ is the weight of the forward-directed HG phase function, and $g_{\mathrm{FWD}}$ and $g_{\mathrm{BKWD}}$ are the asymmetry factors for forward- and backward-directed HG phase functions, respectively. Relationships between $g_{\mathrm{FWD}}, g_{\mathrm{BKWD}}, \alpha$, and $\overline{\cos \theta}$, are provided in [35], [36] and reproduced in the following.

$$
\begin{gathered}
g_{\mathrm{BKWD}}=-0.3061446+1.000568 g_{\mathrm{FWD}}-0.01826338 g_{\mathrm{FWD}}^{2}+0.03643748 g_{\mathrm{FWD}}^{3} \\
\alpha=\frac{g_{\mathrm{BKWD}}\left(1+g_{\mathrm{BKWD}}\right)}{\left(g_{\mathrm{FWD}}+g_{\mathrm{BKWD}}\right)\left(1+g_{\mathrm{BKWD}}-g_{\mathrm{FWD}}\right)} \\
\overline{\cos \theta}=\alpha\left(g_{\mathrm{FWD}}+g_{\mathrm{BKWD}}\right)-g_{\mathrm{BKWD}}
\end{gathered}
$$

In addition, an approximate equation, obtained via regression on the experimental data of [37], is proposed in [36]:

$$
\overline{\cos \theta}=2 \frac{1-2 B}{2+B},
$$

${ }^{2}$ In [33], based on the Petzold's measurements of VSF [34], $g$ is calculated for clean ocean, coastal, and turbid harbor waters. For these three water types, $g$ is equal to $0.8708,0.9470$, and 0.9199 , respectively. We have verified that the small difference between these $g$ values has a negligible effect on the optical channel characteristics, especially on the channel time dispersion. As a result, we take the average value of $g=0.924$ proposed in [12] for all water types. 
where $B=b_{b} / b$. Now, given $b$ and $b_{b}$, using (11)-(14) we calculate the parameters $\overline{\cos \theta}, g_{\mathrm{FWD}}$, $g_{\mathrm{BKWD}}$, and $\alpha$. Then, we generate a RV $\chi_{\mathrm{TTHG}}$ of distribution $\mathcal{U}[0, \pi]$ and use it to calculate the corresponding $\theta$ similar to (9).

We have compared in Fig. 3 the phase functions based on the HG and TTHG photon scattering models for $\overline{\cos \theta}=0.924$. We notice a significant difference between the two phase functions for small and large angles.

\section{NUMERICAL RESULTS}

We provide here some simulation results mainly to study the characteristics of the underwater optical channel. We consider the following typical parameters in our system by default: the wavelength $\lambda=532 \mathrm{~nm}$, a beam width of $w_{0}=3 \mathrm{~mm}$, a maximum beam divergence of $\theta_{0, \max }=$ $20^{\circ}$, a link distance of $Z=20 \mathrm{~m}$, and a receiver aperture diameter of $D=20 \mathrm{~cm}$. Furthermore, we work by default in clear ocean waters with the typical $C$ of $0.31 \mathrm{mg} \cdot \mathrm{m}^{-3}$. We do not consider any spatial filtering at the receiver because in deep-sea waters, in which we are particularly interested, we can effectively neglect background radiations. Therefore, there is usually no need to limit the receiver FOV and this parameter is set to $180^{\circ}$ afterwards.

We will study the impact of the main system parameters on the underlying optical channel, and in particular, on the channel IR.

\section{A. Received intensity as a function of distance}

Let us start by considering the effect of the attenuation coefficient $c$ on the total received intensity that we denote by $I_{r}$. We have shown in Fig. 4 curves of $I_{r}$ as a function of distance $Z$ for the four water types specified in Table I. Results are presented for the HG scattering model with $\overline{\cos \theta}=0.924$, and the TTHG model while calculating $\overline{\cos \theta}$ from (14). ${ }^{3}$

Considering a given water type, we notice a difference between the results corresponding to the two photon scattering models. This difference is mainly due to the better approximation of

\footnotetext{
${ }^{3}$ For instance, for the pure sea water case, we have $b=0.00296 \mathrm{~m}^{-1}$ and $b_{b}=0.00085 \mathrm{~m}^{-1}$, resulting in $B=0.1969$ and $\overline{\cos \theta}=0.3696$. On the other hand, for the clean ocean waters, for instance, we have $b=0.08042 \mathrm{~m}^{-1}$ and $b_{b}=0.00105 \mathrm{~m}^{-1}$, resulting in $B=0.0131$ and $\overline{\cos \theta}=0.967$ [12], [27].
} 
small and large angle photon scattering in the TTHG model, as discussed in Section V and illustrated in Fig.3. It is also due to the difference of the $\overline{\cos \theta}$ for the two cases. In fact, in the TTHG model, $\overline{\cos \theta}$ depends on the water type, unlike the HG model where it is set to 0.924 . In particular, for the case of pure sea waters, we have a large difference between $\overline{\cos \theta}$ by the HG and TTHG models, that leads to a significant difference between the $I_{r}$ curves.

Let us now focus on the results corresponding to the TTHG model. Let us assume a tolerable loss of $100 \mathrm{~dB}$ beyond which the signal is not detectable at the receiver. Note that, in practice, this limit depends on the transmitter power and the receiver sensitivity. With this assumption, we notice from Fig. 4 that the transmission range is limited to $27 \mathrm{~m}, 46 \mathrm{~m}$, and $100 \mathrm{~m}$, for coastal, clear ocean, and pure sea waters, respectively. When working in turbid or estuary waters, on the other hand, the high signal attenuation limits the communication range to less than a few meters. Obviously, the range limit depends on the aperture size, because increasing $D$ allows collecting more scattered photons [1].

Afterwards, the simulation results that we present are obtained based on the TTHG model for photon scattering.

\section{B. Channel impulse response}

The most useful information concerning the channel is its IR using which one can quantify the signal attenuation and the time dispersion. We have conducted four sets of simulations to study the IR for different cases of link distance, receiver lens diameter, transmitter parameters, and water type. To quantify the time dispersion, we define the parameter $\tau$, which is the duration over which the IR falls to $-20 \mathrm{~dB}$ below its peak. We will refer to $\tau$ as the channel dispersion. The main results concerning the study of channel IR presented below, are summarized in Table II.

1) IR for different water types: The IRs for pure sea, clean ocean, and coastal waters are compared in Fig. 5 considering the default values for other system parameters. The case of turbid harbor waters is not represented because too few photons can reach the distance of $Z=20 \mathrm{~m}$ for this water type, as it can also be seen from Fig. 4. The abscissa represents the absolute propagation time from the transmitter to the receiver. We notice that the channel dispersion 
$\tau$ is about $0.21 \mathrm{~ns}, 0.26 \mathrm{~ns}$, and $0.28 \mathrm{~ns}$, for pure sea, clean ocean, and coastal water cases, respectively. So, for typical data-rates (below Gbps), the channel can practically be considered as non-dispersive, and ISI as negligible.

2) Effect of the receiver aperture size: The effect of the receiver lens diameter $D$ on the IR is illustrated in Fig. 6 for the case of clean ocean waters. We have intentionally considered the two extreme cases of $D=0.5 \mathrm{~cm}$ (too small) and $50 \mathrm{~cm}$ (too large) to see clearly the impact on the IR. Obviously, the use of a larger lens leads to a higher photon count: we notice a $22.5 \mathrm{~dB}$ increase in the IR peak by increasing $D$ from $0.5 \mathrm{~cm}$ to $50 \mathrm{~cm}$. Enlarging the receiver lens results also in widening the channel IR since more scattered photons are collected in this way. For instance, $\tau$ is increased from $0.22 \mathrm{~ns}$ to $0.32 \mathrm{~ns}$ by increasing $D$ from $0.5 \mathrm{~cm}$ to $50 \mathrm{~cm}$. We notice that, even using a large lens of $D=50 \mathrm{~cm}$, we practically do not suffer from ISI.

3) Impact of link distance: Figure 7 shows the channel IR for $D=20 \mathrm{~cm}$ three link distances of $Z=10 \mathrm{~m}, 20 \mathrm{~m}$, and $50 \mathrm{~m}$. As expected, channel becomes more dispersive by increasing $Z$. The channel dispersion $\tau$ is about $0.24 \mathrm{~ns}, 0.26 \mathrm{~ns}$, and $0.43 \mathrm{~ns}$ for the three $Z$ values, respectively. The interesting result is that $\tau$ remains negligible even for a relatively long distance of $50 \mathrm{~m}$.

4) Impact of the transmitter parameters: We have investigated the effect of the transmitter beam width $w_{0}$ and the maximum initial beam divergence angle $\theta_{\max }$ on the channel dispersion. We noticed that $w_{0}$ has a negligible impact on $\tau$ unless for a too small receiver aperture (results are not shown for the sake of brevity). The parameter $\theta_{\max }$, however, affects considerably the IR peak since it directly influences channel attenuation. In addition, for a larger $\theta_{\max }$, photons are deviated more from the optical axis, leading to a larger $\tau$. The channel IR parameters are presented in Table II for $\theta_{\max }=0,2^{\circ}$, and $45^{\circ}$.

\section{Bit-error-rate performance}

Finally, to give insight into a practical system implementation, we consider a simple communication system and evaluate its BER performance versus the link distance. We consider the default system parameters defined in the previous subsection as well as the cases of clean ocean and coastal water types. We assume that the transmitter and the receiver are perfectly aligned and time synchronized. We use a high-power $532 \mathrm{~nm}$ LED at the transmitter and a silicon PIN 
photodiode at the receiver with the quantum efficiency of $\eta=16 \%$. We use the simple OOK modulation without any error correcting coding and set the bit rate to 1 Gbps. At the receiver, after photo-detection, the photo-current is converted to a voltage by a trans-impedance circuitry with a resistance $R=1 K \Omega$, and the resulting signal is low-pass filtered to limit the thermal noise variance [38]. Then, we time sample the signal and proceed to signal detection based on optimum thresholding. Note that, since we consider a PIN diode, the dominant noise at the receiver is the thermal noise [38]. In fact, in contrary to atmospheric (free-space) optical communication [39], here the background noise can be neglected because our UWSN has to be deployed in deep waters where the sunlight cannot penetrate.

Figure 8 shows the BER curves as a function of the distance $Z$ for two cases of $P_{t}=0.01 \mathrm{~W}$ and $0.1 \mathrm{~W}$, where $P_{t}$ is the transmit (optical) power. If we consider a required BER of $10^{-6}$, the maximum distances for reliable data transmission is about $18.2 \mathrm{~m}$ and $12.6 \mathrm{~m}$ for $P_{t}=0.1 \mathrm{~W}$, and $30.6 \mathrm{~m}$ and $20.5 \mathrm{~m}$ for $P_{t}=1 \mathrm{~W}$, for the two cases of clear ocean and coastal waters, respectively.

\section{CONCLUSIONS}

We presented in this paper a realistic model for underwater wireless optical channels using an elaborate Monte Carlo simulator. When used for an initial system design, our model is more realistic than the simple Lambert law where neglecting the scattering results in a too optimistic predicted system performance. It is also more accurate than the previously proposed model based on the simple HG phase function. We took different parameters such as the water type and the characteristics of the transmitter and the receiver into account. Through this study, we confirmed that the channel delay dispersion is negligible in most practical cases. Even when working over distances up to $50 \mathrm{~m}$ in clear waters, we showed that the channel can effectively be considered as frequency non-selective. Therefore, we practically do not suffer from any ISI, and do not need to perform computationally complex signal processing such as channel equalization at the receiver.

This work has been an important phase based on which we can now proceed to the next steps of the UWSN design. 


\section{ACKNOWLEDGMENT}

This work has been supported in part by the Marie Curie initial training network related to the FP7 SENSEnet European project. The authors wish to thank Dr. Anabela Dasilva from Institut Fresnel, Marseille, France, for the fruitful discussions on the radiative transfer theory and the Monte Carlo simulation method.

\section{REFERENCES}

[1] C. Gabriel, M. A. Khalighi, S. Bourennane, P. Léon, and V. Rigaud, "Channel modeling for underwater optical communication," IEEE Workshop on Optical Wireless Communications, Global Communication Conference, Dec. 2011, Houston, TX (accepted).

[2] International Sensor Development Network, SENSEnet, http://www.eu-sensenet.net/.

[3] B. M. Cochenour, L. J. Mullen, and A. E. Laux, "Characterization of the beam-spread function for underwater wireless optical communications links," IEEE Journal of Oceanic Engineering, vol. 33, no. 4, pp. 513-521, Oct. 2008.

[4] I. F. Akyildiz, D. Pompili, and T. Melodia, "Underwater acoustic sensor networks: research challenges," Ad Hoc Networks, vol. 3, no. 3, pp. 257-279, 2005.

[5] G. Shah, "A survey on medium access control in underwater acoustic sensor networks," in International Conference Workshops on Advanced Information Networking and Applications (WAINA), May 2009, pp. 1178-1183, Bradford, UK.

[6] F. Pignieri, F. De Rango, F. Veltri, and S. Marano, "Markovian approach to model underwater acoustic channel: Techniques comparison,” in Military Communications Conference (MILCOM), Nov. 2008, pp. 1-7, San Diego, CA.

[7] F. Hanson and S. Radic, "High bandwidth underwater optical communication," Applied Optics, vol. 47, no. 2, pp. 277-283, Jan. 2008.

[8] J. W. Giles and I. Bankman, "Underwater optical communcations systems. Part 2: Basic design considerations," in IEEE Military Communications Conference (MILCOM), Oct. 2005, vol. 3, pp. 1700-1705, Atlantic City, NJ.

[9] D. Anguita, D. Brizzolara, and G. Parodi, "Building an underwater wireless sensor network based on optical communication: research challenges and current results," International Conference on Sensor Technologies and Applications, SENSORCOMM, pp. 476-479, Aug. 2009, Athens, Greece.

[10] S. Arnon, "Underwater optical wireless communication network," Optical Engineering, vol. 49, no. 1, pp. 1-6, Jan. 2010.

[11] S. Jaruwatanadilok, "Underwater wireless optical communication channel modeling and performance evaluation using vector radiative transfer theory," IEEE Journal on Selected Areas in Communications, vol. 26, no. 9, pp. 1620-1627, Dec. 2008.

[12] C. D. Mobley, Light and Water: Radiative Transfer in Natural Waters, Academic Press, June 1994.

[13] J. H. Smart, "Underwater optical communications systems. Part 1: Variability of water optical parameters," in IEEE Military Communications Conference, Oct. 2005, vol. 2, pp. 1140-1146, Atlantic City, NJ.

[14] I. Vasilescu, K. Kotay, D. Rus, M. Dunbabin, and P. Corke, "Data collection, storage, and retrieval with an underwater sensor network," in International Conference on Embedded Networked Sensor Systems (SenSys), Nov. 2005, pp. 154-165, San Diego, CA. 
[15] I. Vasilescu, C. Detweiler, and D. Rus, "Aquanodes: An underwater sensor network,” in Workshop on Underwater networks (WuWNet), MobiCom Conference, Sept. 2007, pp. 85-88, Montreal, Canada.

[16] M. Tivey, P. Fucile, and E. Sichel, "A low power, low cost, underwater optical communication system," Ridge 2000 Events, pp. 27-29, Apr. 2004.

[17] F. Schill, U. R. Zimmer, and J. Trumpf, "Visible spectrum optical communication and distance sensing for underwater applications," Australian Conference on Robotics and Automation (ACRA), Dec. 2004, Canberra, Australia.

[18] M. Doniec, I. Vasilescu, M. Chitre, and C. Detweiler, "AquaOptical: A lightweight device for high-rate long-range underwater point-to-point communication,” IEEE OCEANS Conference, pp. 1-6, Oct. 2009, Biloxi, MS.

[19] J. Lu, S. Lee, J. Mounzer, and C. Schurgers, “Low-cost medium range optical underwater modem,” in ACM International Workshop on UnderWater Networks (WUWNet), Nov. 2009, Berkeley, CA.

[20] C.D. Mobley, B. Gentili, H. R. Gordon, Z. Jin, G. W. Kattawar, A. Morel, P. Reinersman, K. Stamnes, and R. H. Stavn, "Comparison of numerical models for computing underwater light fields," Applied Optics, vol. 32, no. 36, pp. 7484-7504, Dec. 1993.

[21] D. J. Bogucki, J. Piskozub, M.-E. Carr, and G. D. Spiers, "Monte carlo simulation of propagation of a short light beam through turbulent oceanic flow," Optics Express, vol. 15, no. 21, pp. 13988-13996, Oct. 2007.

[22] L. C. Andrews and R. L. Phillips, Laser Beam Propagation Through Random Media, SPIE Press, second edition, 2005.

[23] M. A. Khalighi, N. Schwartz, N. Aitamer, and S. Bourennane, "Fading reduction by aperture averaging and spatial diversity in optical wireless systems," IEEE/OSA Journal of Optical Communications and Networking, vol. 1, no. 6, pp. 580-593, Nov. 2009.

[24] J. A. Simpson, B. L. Hughes, and J. F. Muth, "A spatial diversity system to measure optical fading in an underwater communications channel," in IEEE OCEANS Conference, Oct. 2009, pp. 1-6, Biloxi, MS.

[25] F. Hanson and M. Lasher, "Effects of underwater turbulence on laser beam propagation and coupling into single-mode optical fiber," Applied Optics, vol. 49, no. 16, pp. 3224-3230, June 2010.

[26] C. F. Bohren and D. R. Huffman, Absorption and Scattering of Light by Small Particles, Wiley, 1988.

[27] V. I. Haltrin, "Chlorophyll-based model of seawater optical properties," Applied Optics, vol. 38, no. 33, pp. 6826-6832, Nov. 1999.

[28] V. I. Haltrin and G. W. Kattawar, "Self-consistent solutions to the equation of transfer with elastic and inelastic scattering in oceanic optics: I. Model," Applied Optics, vol. 32, no. 27, pp. 5356-5367, 1993.

[29] G. Kervern, "Lidars sous-marins," Techniques de l’Ingénieur, vol. 6, no. E4325, pp. 1-7, Nov. 1997.

[30] A. Ishimaru, Wave Propagation and Scattering in Random Media, IEEE Press, 1997.

[31] L. Wang, S. L. Jacques, and L. Zheng, "MCML, Monte Carlo modeling of light transport in multi-layered tissues," Tech. Rep., Laser Biology Research Laboratory, Nov. 1995, University of Texas, M.D. Anderson Cancer Center.

[32] H. C. van de Hulst, Light Scattering by Small Particles, Dover Publications, 1981.

[33] Yu I. Kopilevich, M. E. Kononenko, and E. I. Zadorozhnaya, "The effect of the forward-scattering index on the characteristics of a light beam in sea water," Journal of Optical Technology, vol. 77, no. 10, pp. 598-601, Oct. 2010.

[34] T. J. Petzold, "Volume scattering functions for selected ocean waters," Technical Report SIO 7278, Scripps Institute of Oceanography, 1972. 
[35] V. Haltrin, "One-parameter two-term henyey-greenstein phase function for ligth scattering in seawater," Applied Optics, vol. 41, no. 6, pp. 1022-1028, Feb. 2002.

[36] V. I. Haltrin, "Two-term henyey-greenstein light scattering phase function for seawater," International Geoscience and Remote Sensing Symposium (IGARSS), pp. 1423-1425, June-July 1999, Hamburg, Germany.

[37] V. Timofeyeva, "Relation between light-field parameters and between scattering phase function characteristics of turbid media, including seawater," Ocean Physics, vol. 14, pp. 834-848.

[38] F. Xu, M. A. Khalighi, and S. Bourennane, "Impact of different noise sources on the performance of PIN- and APD-based FSO receivers," COST IC0802 Workshop, IEEE ConTEL Conference, pp. 279-286, June 2011, Graz, Austria.

[39] M. A. Khalighi, F. Xu, Y. Jaafar, and S. Bourennane, "Double-laser differential signaling for reducing the effect of background radiation in free-space optical systems," IEEE/OSA Journal of Optical Communications and Networking, vol. 3, no. 2, pp. 145-154, Feb. 2011. 
TABLE I

ABSORPTION, SCATTERING, BACK SCATTERING, AND ATTENUATION COEFFICIENTS FOR THE FOUR WATER TYPES CONSIDERING TYPICAL CHLOROPHYLL CONCENTRATIONS.

\begin{tabular}{|c|c|c|c|c|c|}
\hline Water type & $C\left(\mathrm{mg} / \mathrm{m}^{3}\right)$ & $a\left(\mathrm{~m}^{-1}\right.$ & $b\left(\mathrm{~m}^{-1}\right)$ & $b_{b}\left(\mathrm{~m}^{-1}\right)$ & $c\left(\mathrm{~m}^{-1}\right)$ \\
\hline \hline Pure sea & 0.005 & 0.053 & 0.003 & 0.0006 & 0.056 \\
\hline Clear ocean & 0.31 & 0.069 & 0.08 & 0.0010 & 0.15 \\
\hline Coastal & 0.83 & 0.088 & 0.216 & 0.0014 & 0.305 \\
\hline Harbor & 5.9 & 0.295 & 1.875 & 0.0076 & 2.17 \\
\hline
\end{tabular}

TABLE II

SUMMARY OF INTENSITY LOSS AND CHANNEL TIME DISPERSION FOR DIFFERENT SYSTEM AND CHANNEL PARAMETERS.

(THE DEFAUlT CASE APPEARS IN BLUE)

\begin{tabular}{|c|c|c|c||c|c|}
\hline$c\left(\mathrm{~m}^{-1}\right)$ & $Z(\mathrm{~m})$ & $D(\mathrm{~cm})$ & $\theta_{\max }\left({ }^{\circ}\right)$ & Intensity loss $(\mathrm{dB})$ & $\tau(\mathrm{ns})$ \\
\hline \hline $\mathbf{0 . 1 5}$ & $\mathbf{2 0}$ & $\mathbf{2 0}$ & $\mathbf{2 0}$ & $-\mathbf{3 0 . 4 1}$ & $\mathbf{0 . 2 6}$ \\
\hline \hline $\mathbf{0 . 0 5 6}$ & 20 & 20 & 20 & -23.50 & 0.21 \\
\hline $\mathbf{0 . 3 0 5}$ & 20 & 20 & 20 & -39.74 & 0.28 \\
\hline \hline 0.15 & 20 & $\mathbf{0 . 5}$ & 20 & -48.38 & 0.22 \\
\hline 0.15 & 20 & $\mathbf{2}$ & 20 & -41.44 & 0.24 \\
\hline 0.15 & 20 & $\mathbf{5 0}$ & 20 & -25.89 & 0.32 \\
\hline \hline 0.15 & $\mathbf{1 0}$ & 20 & 20 & -21.23 & 0.24 \\
\hline 0.15 & $\mathbf{5 0}$ & 20 & 20 & -53.52 & 0.43 \\
\hline \hline 0.15 & 20 & 20 & $\mathbf{0}$ & -12.28 & 0.17 \\
\hline 0.15 & 20 & 20 & $\mathbf{2}$ & -20.46 & 0.22 \\
\hline 0.15 & 20 & 20 & $\mathbf{4 5}$ & -33.94 & 0.27 \\
\hline
\end{tabular}




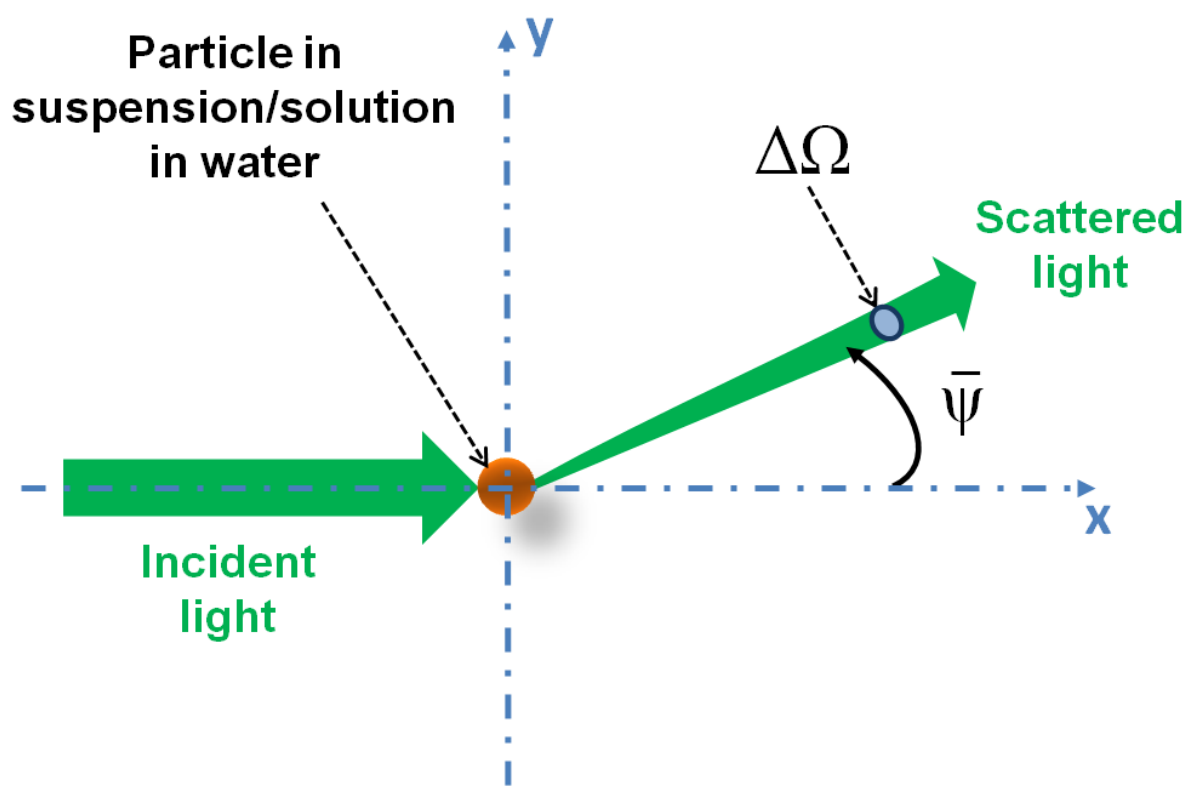

Fig. 1. Light scattering when encountering a particle in water. Part of the incident light flux is absorbed by the particle and the remaining flux is scattered through an angle $\Psi$. The scattering direction $\Psi$ is within a solid angle $\Delta \Omega$ around $\bar{\Psi}$. 


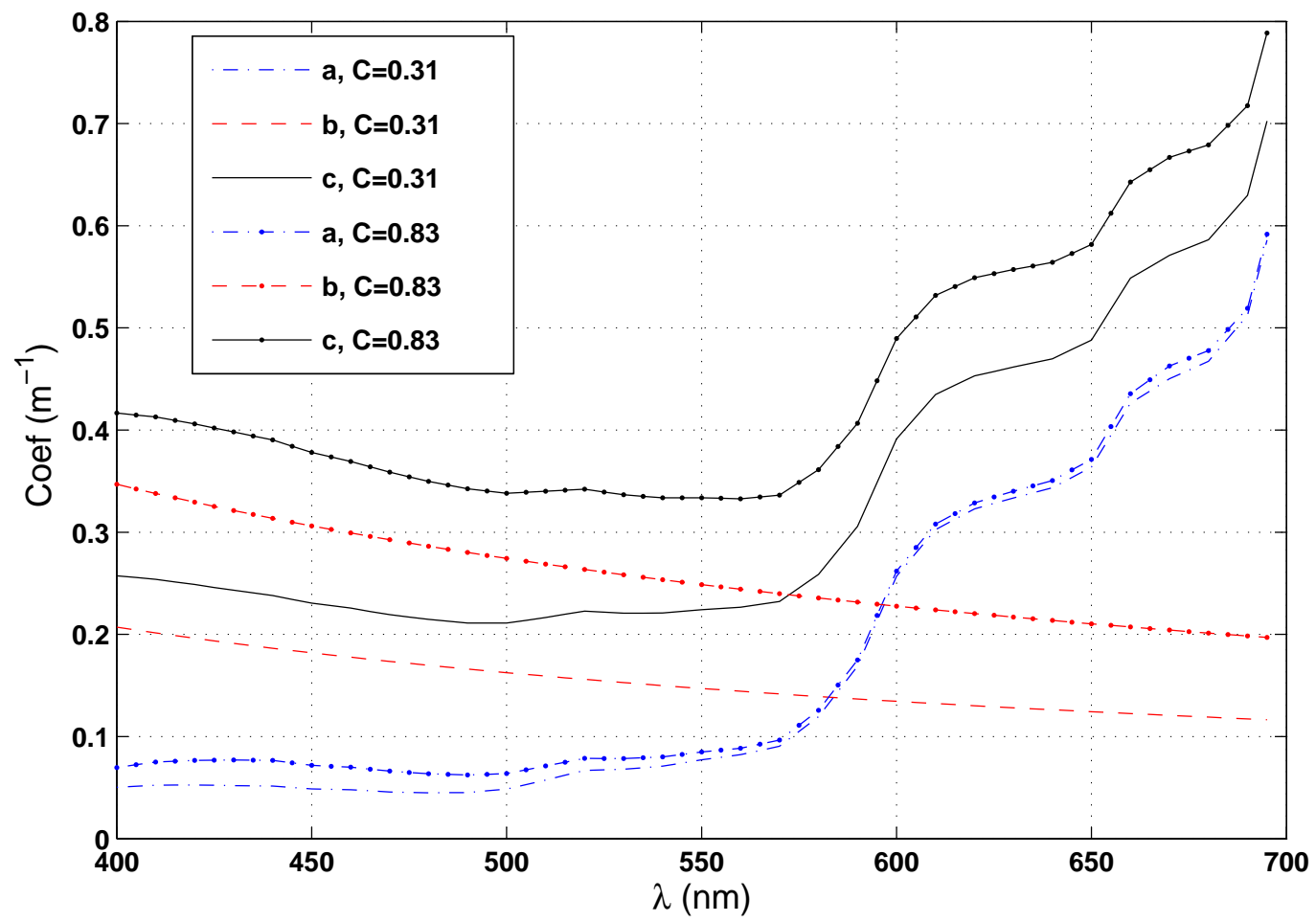

Fig. 2. Absorption $a$, scattering $b$, and extinction $c$ coefficients as a function of the wavelength $\lambda$ for two chlorophyll concentrations $C$ (in mg.m ${ }^{-3}$ ) using the model of Gordon and Morel [12]. 


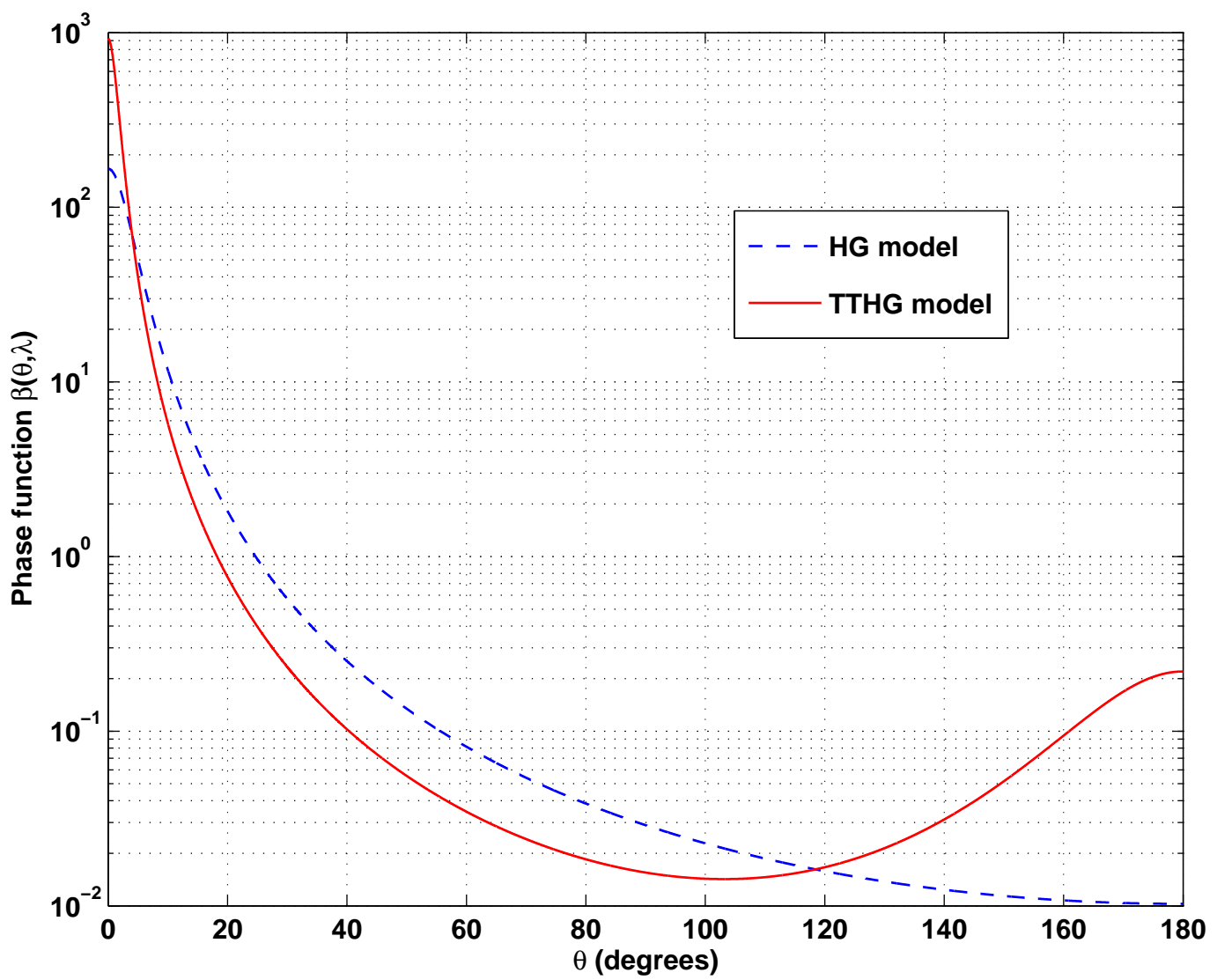

Fig. 3. Comparison between HG and TTHG phase functions for $\overline{\cos \theta}=0.924$. 


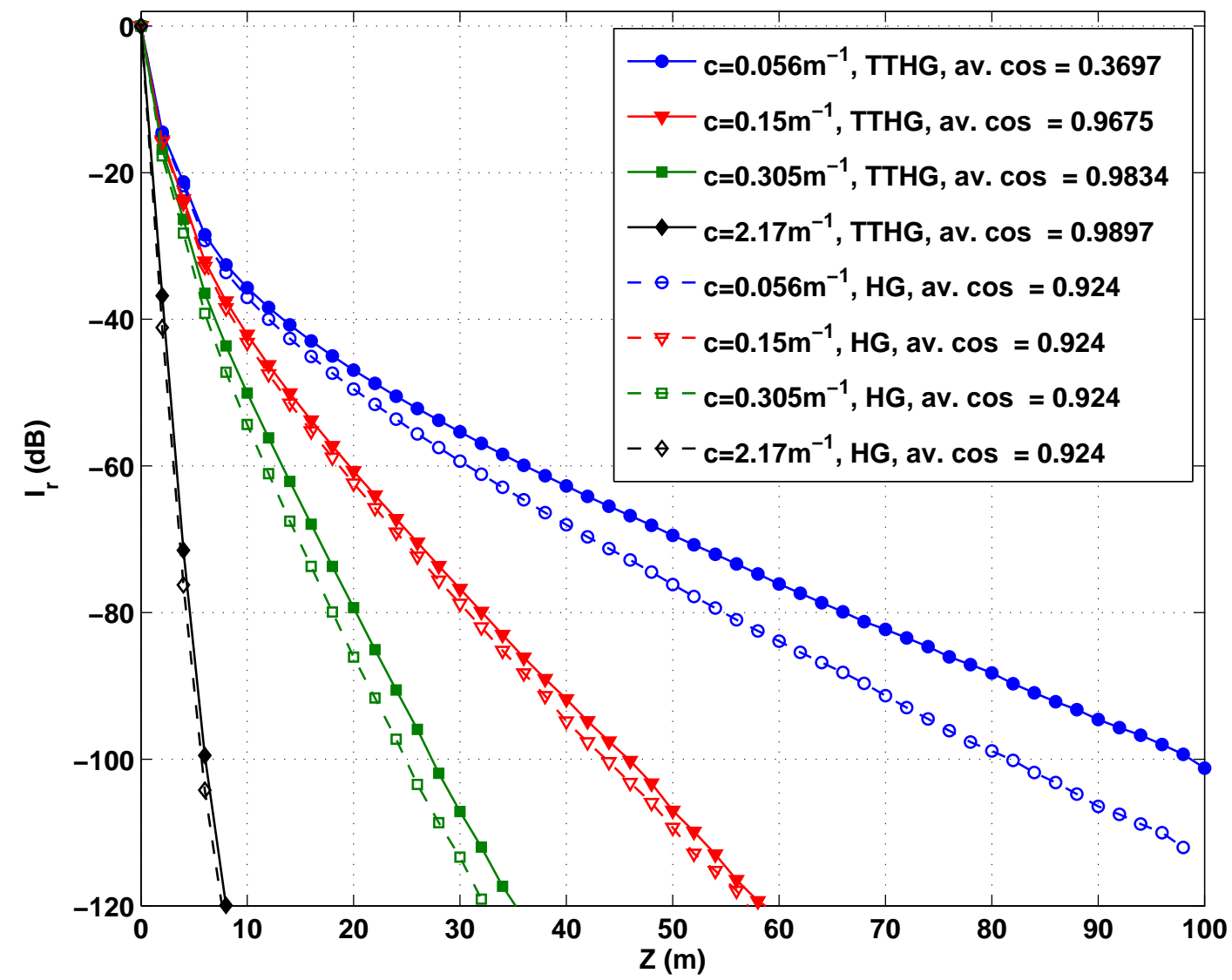

Fig. 4. Received intensity (in $\mathrm{dB}$ ) as a function of distance for different water types, $D=20 \mathrm{~cm}$. "av. $\cos$ " denotes $\overline{\cos \theta}$. 


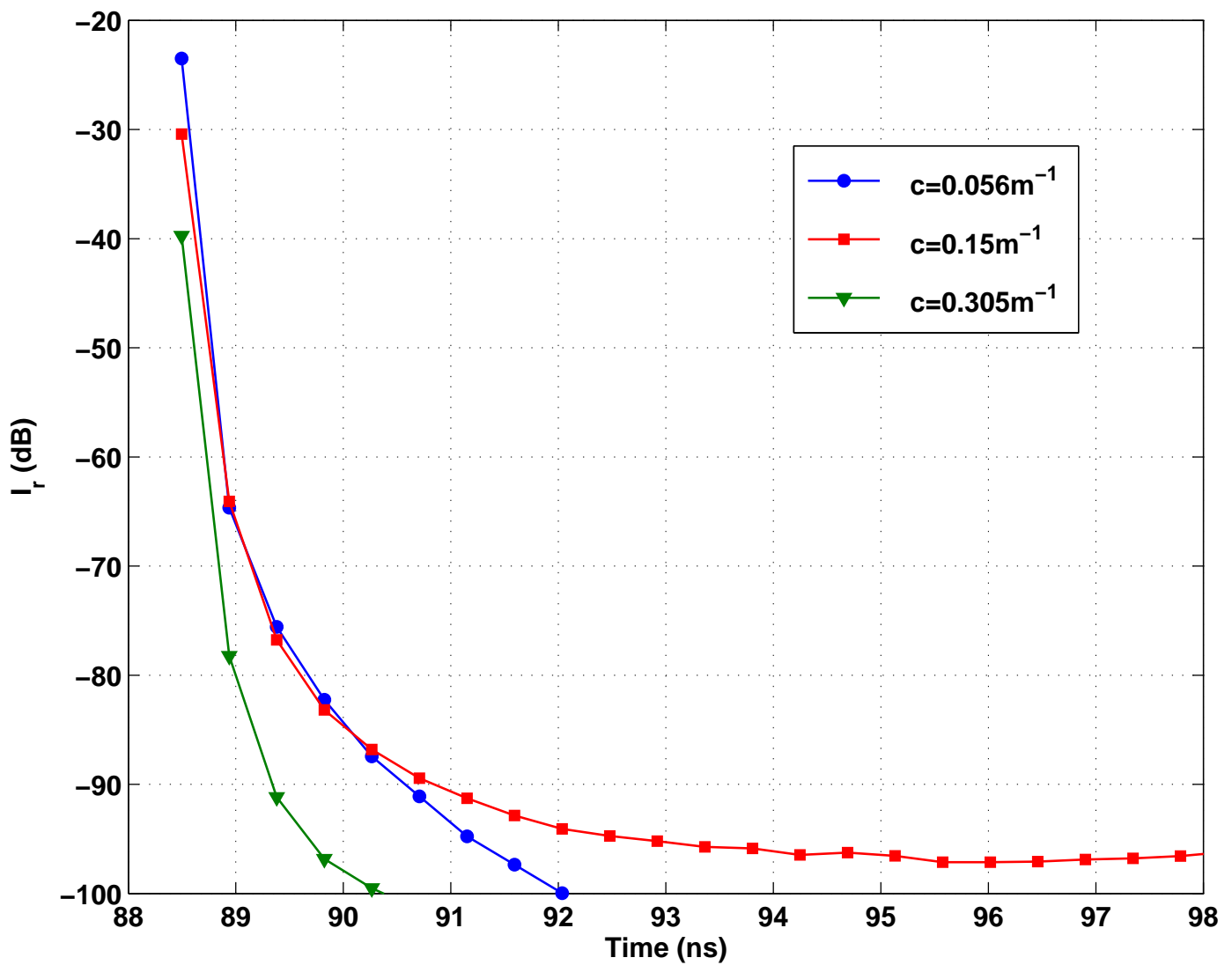

Fig. 5. Channel IR (received intensity as a function of time) for pure sea and clean ocean waters. $Z=20 \mathrm{~m}, D=20 \mathrm{~cm}$ 


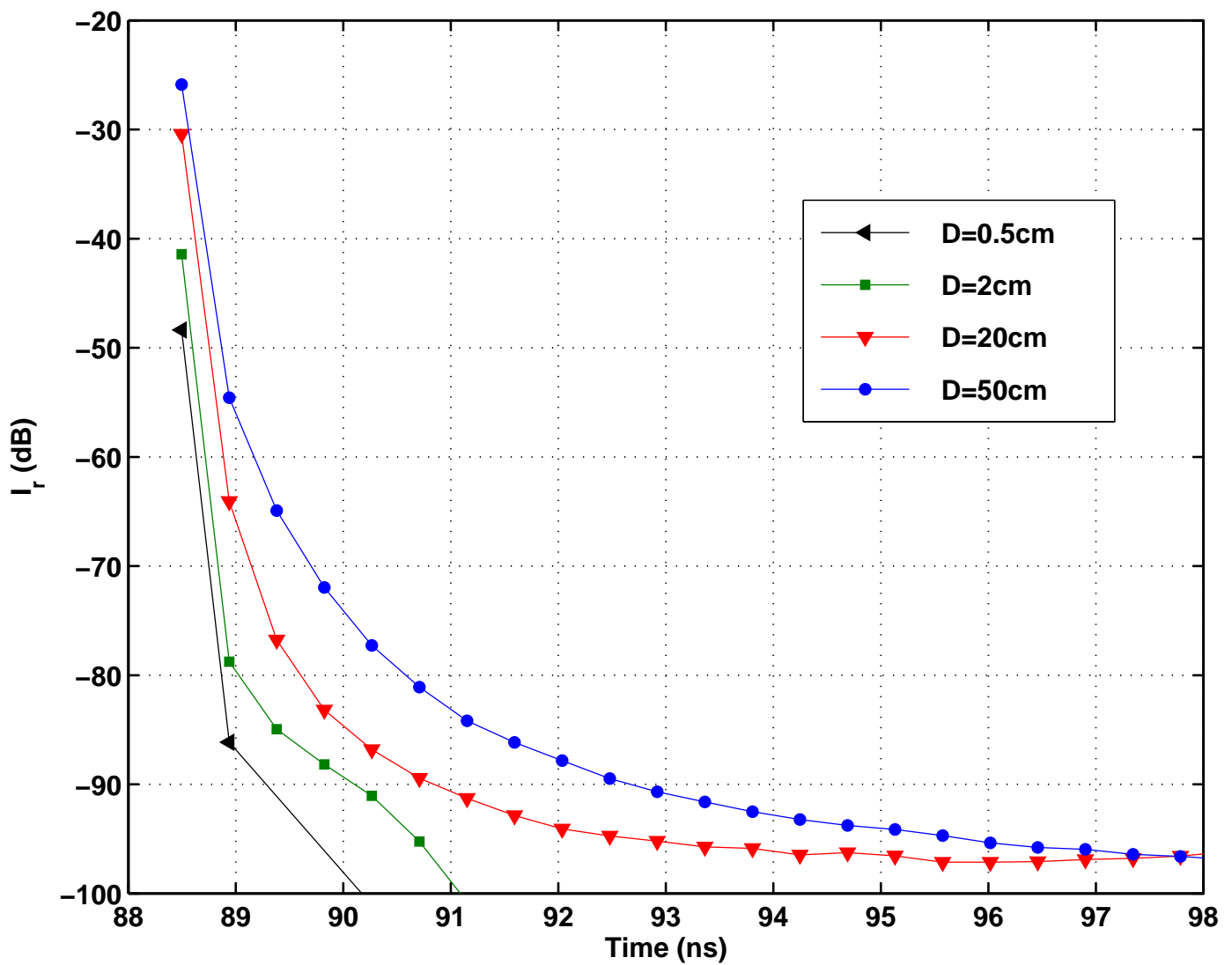

Fig. 6. Received intensity as a function of aperature diameter D. $Z=20 \mathrm{~m}$, clear ocean waters. 


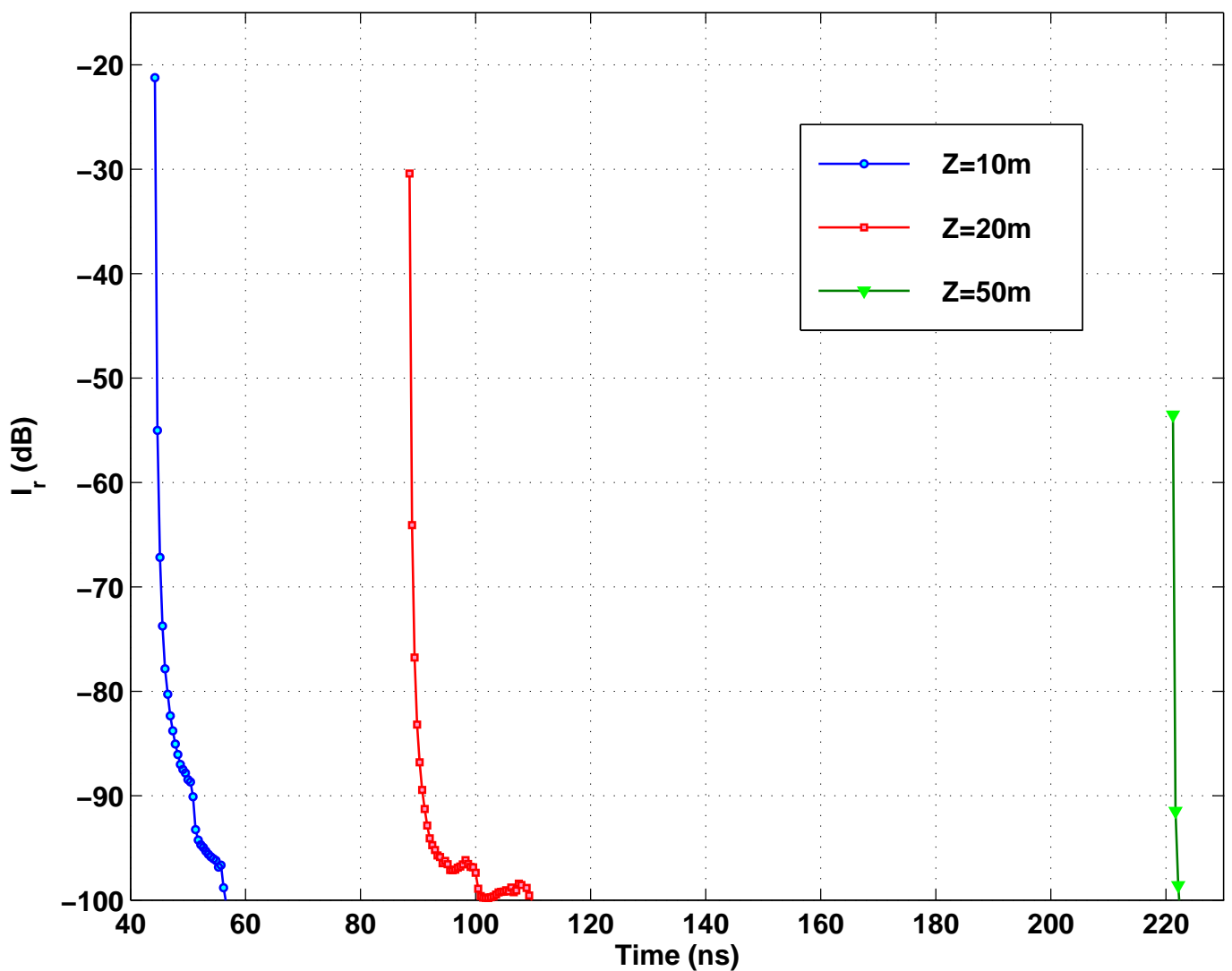

Fig. 7. Received intensity as a function of link distance $Z . D=20 \mathrm{~cm}$, clear ocean waters. 


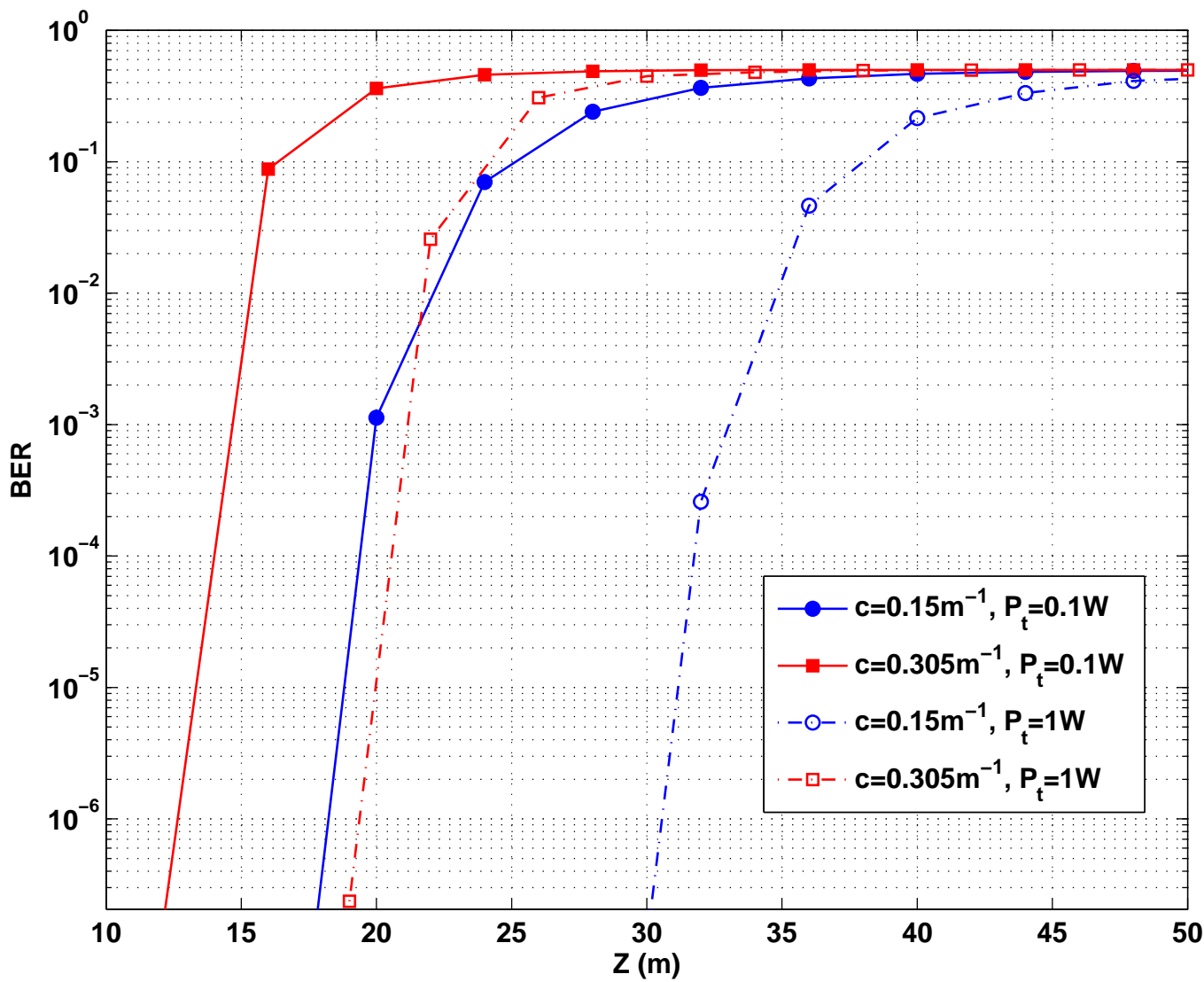

Fig. 8. BER performance in function of the distance $Z$ for different transmit optical powers $P_{t}$. Clear ocean and coastal waters, $D=20 \mathrm{~cm}$. 